ABSTRACT: Obese individuals have slowed conduction in the median nerve across the wrist, but the mechanism for this is not established. This case-control study of 27 obese subjects and 16 thin subjects was designed to test the hypothesis that obese individuals have higher carpal canal pressures and more median nerve swelling than thin individuals. All subjects were asymptomatic for hand symptoms, and had measurements of median and ulnar sensory nerve conduction in the nondominant hand, ultrasound measurement of the median nerve cross-sectional area proximal to the carpal canal, and carpal canal pressure measurement. There was no difference in age or gender ratio between the obese and thin groups. The median nerve cross-sectional area was equal in the obese and thin groups $\left(9.3 \mathrm{~mm}^{2}\right.$ vs. $9.4 \mathrm{~mm}^{2}$ ), as was the carpal canal pressure $(16.2 \mathrm{mmHg}$ vs. $15.5 \mathrm{mmHg}$, respectively). There was a strong correlation between median nerve conduction slowing across the wrist and median nerve cross-sectional area at the wrist $(r=0.55, P=0.002)$. Obesity does not influence carpal canal pressure or the size of the median nerve at the wrist. However, there is a strong association between slowed median nerve conduction and increased nerve size which suggests endoneurial edema as a metabolic mechanism; the conduction slowing does not appear to be related to mechanical stress.

Muscle Nerve 30: 481-485, 2004

\title{
INFLUENCE OF BODY MASS INDEX ON MEDIAN Nerve function, CARPAL CANAL PRESSURE, AND CROSS-SECTIONAL AREA OF THE MEDIAN NERVE
}

\author{
ROBERT A. WERNER, MD, , ${ }^{1,2}$ JON A. JACOBSON, MD, ${ }^{3}$ and DAVID A. JAMADAR, MB, BS ${ }^{3}$ \\ ${ }^{1}$ Department of Physical Medicine and Rehabilitation, University of Michigan Medical Center, \\ Ann Arbor, Michigan, USA \\ ${ }^{2}$ Physical Medicine and Rehabilitation Service, Veterans Affairs Medical Center, 2215 Fuller Road, \\ Ann Arbor, Michigan 48105-2203, USA \\ ${ }^{3}$ Department of Radiology, University of Michigan Medical Center, Ann Arbor, Michigan, USA
}

Accepted 28 May 2004

Several investigators have demonstrated that carpal tunnel syndrome (CTS) is more prevalent among heavier and shorter persons in the general population. $2,3,4,10,13$ Persons classified as obese are two to three times more likely to be diagnosed with CTS. ${ }^{2,4,13}$ Within industrial populations, the relative risk is four times higher for obese workers to develop CTS than thin workers; the more obese the subject, the higher is the risk for CTS. ${ }^{9,15,16}$

The pathophysiologic basis of this relationship is not well understood. Letz and Gerr ${ }^{8}$ found the same relationship between obesity and slow conduction of the median nerve across the wrist in a large cross-

\footnotetext{
Abbreviations: BMI, body mass index; CTS, carpal tunnel syndrome Key words: carpal tunnel syndrome; electromyography; median nerve; pressure; ultrasound

Correspondence to: R. A. Werner; e-mail: rawerner@umich.edu

(C) 2004 Wiley Periodicals, Inc.

Published online 4 August 2004 in Wiley InterScience (www.interscience. wiley.com). DOI 10.1002/mus.20125
}

sectional study, but an inverse relationship was found between obesity and other peripheral nerve measures. Conduction velocity of the peroneal, sural, and ulnar nerves all tended to improve among subjects who were more obese, and only the median sensory nerve across the wrist demonstrated slowing. The differential nerve responses to obesity suggest that the carpal tunnel has a unique influence on the median nerve in response to increasing body mass index (BMI).

Although there is a strong relationship between obesity and slowing of median nerve conduction across the wrist, obesity alone does not predict whether an individual develops CTS. When statistical models were developed to predict who would develop CTS, obesity explained only a small portion of the variance (less than $8 \%$ ) related to the diagnosis of CTS or electrodiagnostic abnormalities involving the median nerve. ${ }^{9,16}$

The interstitial fluid pressure within the carpal tunnel is higher among patients with CTS, ${ }^{5,14,17}$ and 
patients with CTS have swelling of the median nerve that is most prominent just proximal to the carpal canal. ${ }^{1}$ The present study was conducted to explore whether obesity influences median nerve function and, if so, whether this is associated with changes in the carpal canal hydrostatic pressure or the anatomy of the median nerve as demonstrated by ultrasound imaging. We hypothesized that obese subjects would: (1) have relative prolongation of the median sensory latency compared to the ulnar sensory latency, (2) have higher carpal canal pressures, and (3) have swelling of the median nerve proximal to the carpal tunnel.

\section{METHODS}

This was a prospective, case-control study of asymptomatic subjects comparing thin and obese individuals. Obese was defined as a BMI of over 30, and thin was defined as a BMI of less than 25. We recruited 43 subjects for the study, of whom 16 were thin and 27 were obese. Subjects were recruited from the general population through newspaper advertisements. The project was approved by our local institutional review board.

To be included, subjects were required to be between 18 and 75 years old and to have no complaints of hand or wrist discomfort. Subjects also had to meet the BMI criteria noted above and to have no history of diabetes, thyroid disease, arthritis, upperextremity fracture, or CTS.

Subjects were weighed (without shoes) and height was measured. Each subject filled out a brief questionnaire with work history, estimate of hand work intensity, and exercise level. Hand work intensity and exercise were based upon self-report using a 5-point Likert scale. Sensory conduction studies of the median and ulnar nerves, and measurement of the cross-sectional area of the median nerve and hydrostatic fluid measurement within the carpal canal were performed in the nondominant hand, which was chosen to minimize the influence of handintensive activity that occurs in the dominant hand.

To measure the intracarpal canal pressures, the volar surface of the wrist and the carpal canal were anesthetized. A hollow 18-gauge pressure-measuring needle (Stryker, Kalamazoo, Michigan) was inserted through the volar skin of the wrist, $1 \mathrm{~cm}$ proximal to the distal wrist crease, into the carpal canal at a $45^{\circ}$ angle. The needle was attached to an external pressure transducer and the system was flushed with 0.3 cc of normal saline. The pressure was recorded after passively extending and then flexing the fingers. The interstitial fluid pressures were measured with the wrist in a neutral position and fingers in a relaxed posture. The pressure measurement was repeated three times. The lowest pressure after the fingers were passively extended and flexed was recorded. The average of the three trials was used as the hydrostatic fluid pressure.

Nerve conduction studies of the median and ulnar sensory nerves were performed as described by Kimura, ${ }^{7}$ using antidromic supramaximal stimulation, a distance of $14 \mathrm{~cm}$, and ring recording electrodes placed around digits 2 and 5. A standard interelectrode distance of $3 \mathrm{~cm}$ was used. Hand temperature was recorded and the hand was warmed if the midpalmar temperature was below $32^{\circ} \mathrm{C}$. All studies were performed on-site by a certified electrodiagnostic technician on a Synergy system (TecaOxford Instruments, Pleasantville, New York). The difference in peak sensory latencies was used as the primary electrodiagnostic outcome measure.

Sonographic evaluation of the medial nerve was performed by one of two musculoskeletal radiologists (J.A.J. and D.A.J.) with musculoskeletal sonography experience (10 years and 7 years, respectively) using 12 and $15 \mathrm{MHz}$ linear transducers (Model HDI 5000; Phillips ATL, Bothell, Washington). Liberal transmission gel was used in place of stand-off pad. The median nerve proximal to the carpal tunnel at the level of the volar wrist crease was identified in the transverse plane by its characteristic speckled appearance from hypoechoic nerve fascicles and hyperechoic connective tissue. ${ }^{6}$ Using the circumference trace method with cursor and track ball, the area of the median nerve was measured on the static sonographic image during real-time examination. The outer margin of the median nerve was traced at the junction of the hypoechoic nerve fascicles and adjacent hyperechoic outer connective tissue layer or epineurium. Transducer orientation was perpendicular to the longitudinal axis of the median nerve during assessment. The median nerve was similarly measured within the carpal tunnel at a level $1 \mathrm{~cm}$ distal to the wrist crease.

\section{STATISTICAL ANALYSIS}

A two-tailed Student's $t$-test was performed to test the mean between the thin and obese subjects for all the outcome and demographic measures, i.e., age, smoking history (pack years), exercise level, hand activity level, median-ulnar sensory latency difference, carpal canal pressure, and cross-sectional area of the median nerve. A chi-squared analysis was done for categorical data such as gender, exercise level, and hand activity level. A pair-wise correlation was done 
on all the outcome measures. A regression model was performed using median-ulnar sensory latency difference as the dependent variable, and BMI, cross-sectional area of the median nerve, carpal canal pressure, gender, and age as the independent variable. All statistics were run on Stata 5.0 (College Park, Texas)

\section{RESULTS}

Table 1 shows the demographic characteristics of the two groups. Recruitment was stratified by age and gender. There were no significant differences between the two groups for age, gender, or hand activity level, but exercise level was significantly different: the thin subjects reported exercising more frequently.

Obese subjects had the same carpal canal pressure as thin subjects and their median nerve crosssectional areas were equivalent (Table 1). The median sensory latency was more prolonged among obese subjects, as has been demonstrated in other studies. $^{8,9,15}$ Obese subjects had a longer medianulnar peak latency difference than controls $(P=$ 0.03 ; Table 1$)$. The population average latency difference is $0.2 \mathrm{~ms}$ for this age group. ${ }^{7}$ All subjects were asymptomatic, but $41 \%$ of obese subjects had a median-ulnar difference that met criteria for a median mononeuropathy compared to $12.5 \%$ of control subjects $(P=0.03)$.

No significant correlation was found between BMI and the cross-sectional area of the median nerve $(r=0.07, P=0.7)$, or between BMI and carpal canal pressure $(r=0.19, P=0.2)$. However, there was a strong correlation between the prolongation of the median sensory peak latency and the cross-sectional area of the median nerve within, and proximal to, the carpal canal ( $r=0.55, P=0.0002$; Fig. 1$)$, and with carpal canal pressure $(r=0.33, P=0.03)$. Smoking and exercise did not have a significant correlation with any of the outcome measures.

Regression analysis yielded a strong model predicting the median-ulnar sensory peak latency difference. The model included BMI, cross-sectional area of the median nerve, carpal canal pressure, gender, and age. The model had an $R^{2}$ value of 0.62 (Prob $>F=0.008$ ). The final regression model is shown below:

$$
\begin{aligned}
& \text { Median-ulnar latency difference }= \\
& \quad-2.8+0.03(\mathrm{BMI})+0.13(\text { median CSA }) \\
& +0.16(\mathrm{CCP})+0.24 \text { (gender) }+0.004 \text { (age) }
\end{aligned}
$$

where median CSA is cross-sectional area of the median nerve and CCP represents carpal canal pressure.

\section{DISCUSSION}

The original hypothesis was incorrect; obesity did not influence carpal canal pressure or the size of the median nerve at the wrist. The asymptomatic, obese subjects did have significant slowing of the median nerve across the wrist, whereas the ulnar nerve conduction was the same in both groups. If the mechanism of action was mechanical, we would have expected thickening of the synovial sheaths of the finger flexor tendons and associated swelling of the median nerve with the related elevated carpal canal pressures. Thickening of the synovial sheaths is the proposed mechanism in many instances of idio-

\begin{tabular}{|c|c|c|c|}
\hline Variables & $\begin{array}{c}\text { Thin } \\
(N=16)\end{array}$ & $\begin{array}{c}\text { Obese } \\
(N=27)\end{array}$ & $\begin{array}{c}\text { Comparison } \\
\text { (P-value })\end{array}$ \\
\hline Age & $52.2 \pm 14.5$ & $53.5 \pm 15.3$ & 0.79 \\
\hline Gender (\% female) & 69 & 67 & 0.89 \\
\hline Body mass index & $22.5 \pm 2.1$ & $35.5 \pm 5.8$ & $<0.001$ \\
\hline Smoking (pack-years) & $0.6 \pm 1.8$ & $2.3 \pm 17.4$ & 0.22 \\
\hline Exercise level ( 1 = low, 5 = high) & $3.7 \pm 0.6$ & $2.3 \pm 1.0$ & $<0.001$ \\
\hline Intensity of hand activities ( $1=$ low, $5=$ high) & $3.4 \pm 0.5$ & $3.3 \pm 1.1$ & 0.59 \\
\hline Median sensory peak latency (ms) & $3.29 \pm 0.47$ & $3.53 \pm 0.58$ & 0.17 \\
\hline Ulnar sensory peak latency (ms) & $3.18 \pm 0.30$ & $3.04 \pm 0.24$ & 0.11 \\
\hline Median-ulnar peak sensory latency difference (ms) & $0.12 \pm 0.32$ & $0.49 \pm 0.62$ & 0.03 \\
\hline Median mononeuropathy (\% abnormal), (median-ulnar latency $\geq 0.5 \mathrm{~ms}$ ) & 12.5 & 40.7 & 0.03 \\
\hline Carpal canal pressure (mmHg) & $15.5 \pm 6.7$ & $16.2 \pm 8.8$ & 0.76 \\
\hline Cross-sectional area of median nerve proximal to carpal canal $\left(\mathrm{mm}^{2}\right)$ & $9.4 \pm 1.5$ & $9.3 \pm 2.4$ & 0.89 \\
\hline Cross-sectional area of median nerve in the carpal canal $\left(\mathrm{mm}^{2}\right)$ & $8.9 \pm 1.6$ & $10.2 \pm 3.8$ & 0.23 \\
\hline
\end{tabular}
pathic CTS where friction of the finger flexor ten-

Table 1. Comparison of thin and obese subjects (mean \pm SD). 


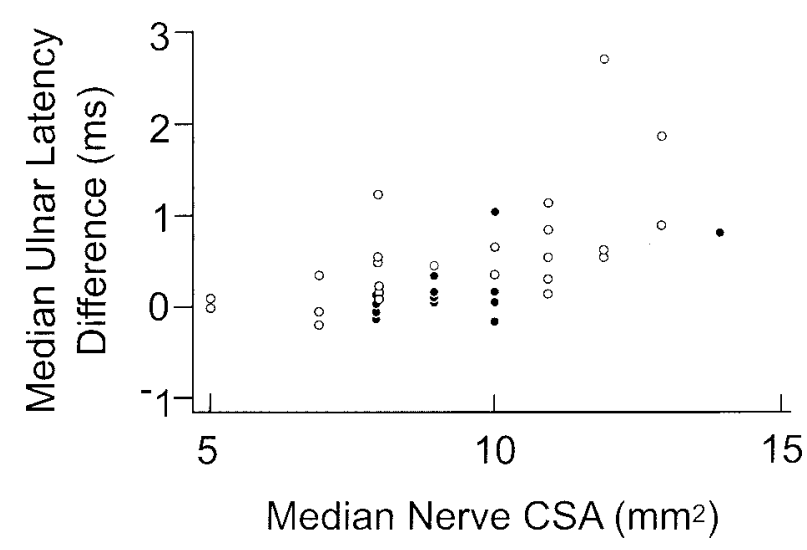

FIGURE 1. Median-ulnar latency difference in relation to median nerve cross-sectional area (CSA) proximal to the carpal canal. Thin subjects are represented by a closed circle and obese subjects are represented by an open circle.

dons over the fulcrum of the bony edges of the carpal canal results in thickening of the synovium with time. This mechanism would explain the increased pressure within the carpal canal and the proximal swelling of the median nerve as it enters the carpal canal. ${ }^{17}$

This study demonstrates that slowing of median nerve conduction across the wrist among asymptomatic subjects is strongly associated with size of the nerve. This relationship has been demonstrated in studies comparing CTS subjects with healthy controls but not among a cohort of healthy, asymptomatic subjects. ${ }^{1}$ Subjects with CTS have swelling of the median nerve proximal to the carpal canal and prolongation of the median nerve response across the wrist. The finding of median nerve swelling is a useful tool in the diagnosis of CTS, ${ }^{1}$ but it is not clear why this relationship should exist among asymptomatic subjects. There was wide variation in the distribution of median nerve sensory latencies, and this was an expected finding among subjects with a high BMI. However, subjects with median nerve conduction slowing were more likely to have swelling of the median nerve; this was not associated with the diagnosis of CTS. The association may represent localized, intrafascicular swelling of the median nerve within the carpal tunnel that is directly related to impairment of nerve conduction. This does not appear to be the same phenomenon that is thought to cause nerve swelling associated with CTS. In CTS, the explanation for the proximal swelling is increased pressure within the carpal tunnel leading to a slowing of axonal transport and localized swelling most prominent proximal to the carpal tunnel. ${ }^{1} \mathrm{An}$ imal studies have demonstrated proximal nerve swelling in response to cuff compression and this lends further support to a mechanical mechanism. ${ }^{11}$ In our study, carpal pressure was not increased, and subjects were asymptomatic. This suggests some type of localized metabolic problem that causes intrafascicular swelling without mechanical interference. Another possibility is that the change in pressure is a transient phenomenon that is affected by posture or activity level and not captured by the single pressure measurement used in the study design.

The predictive model for identifying the relative slowing of median nerve conduction is very strong for a biological system. The age, gender, BMI, crosssectional area of the median nerve, and carpal canal pressure explained $62 \%$ of the variance in our cohort of asymptomatic individuals. There have been other predictive models using age, gender, BMI, and wrist dimensions to predict conduction slowing of the median nerve across the wrist, but these were developed among symptomatic patients screened for CTS. ${ }^{12}$

Given the strong association between median sensory nerve latencies and BMI, the electromyographer should use caution when interpreting nerve conduction studies of obese subjects referred for evaluation of CTS. Our findings, that over $40 \%$ of asymptomatic obese subjects met criteria for a median mononeuropathy, suggest that there is a strong likelihood of a false-positive study when evaluating an obese subject with a questionable clinical presentation for CTS. If the obese subject does not have classic clinical findings of CTS, we suggest the nerve conduction findings be viewed cautiously.

The relationship between obesity and slowing of median sensory conduction across the wrist remains unclear. Our study suggests that increased interstitial pressure is not the primary reason for impairment of the median nerve. It does not exclude transient mechanical forces as a factor, but the mechanism appears to be different from typical CTS where high pressures and synovial thickening are usually demonstrated. The inference is that this is related to a metabolic change. An association between obesity and impaired glucose metabolism is the most likely pathophysiology to explain the occurrence of median nerve impairment among obese individuals, but further research is needed to establish this relationship. Alternatively, a low BMI may be a surrogate for better general health and this may be protective of median sensory nerve function. It remains to be determined why the median nerve becomes swollen and how this affects nerve function.

This work was presented in part at the annual meeting of the American Association of Electrodiagnostic Medicine in San Francisco, California, September 2003. Support for this research was 
provided by the National Institute on Disability and Rehabilitation Research of the United States Department of Education, grant H133E980007: "Rehabilitation Engineering Research Center." The opinions contained in this publication are those of the grantee and do not necessarily reflect those of the United States Department of Education.

\section{REFERENCES}

1. Beekman R, Visser LH. Sonography in the diagnosis of carpal tunnel syndrome: a critical review of the literature. Muscle Nerve 2003;27:26-33.

2. Cannon LJ, Bernacki EJ, Walter DS. Personal and occupational factors associated with carpal tunnel syndrome. J Occup Med 1982;23:255-258.

3. de Krom MC, Kester AD, Knipschild PG, Spann, F. Risk factors for carpal tunnel syndrome. Am J Epidemiol 1990;132: 1102-1110.

4. Dieck GS, Kelsey JL. An epidemiologic study of the carpal tunnel syndrome in an adult female population. Prev Med 1985;14:63-69.

5. Gelberman RH, Rydevik BL, Pess GM, Szabo RM, Lundborg G. Carpal tunnel syndrome. Orthop Clin North Am 1988;19: $115-124$.

6. Jamadar DA, Jacobson JA, Hayes CW. Sonographic evaluation of the median nerve at the wrist. J Ultrasound Med 2001;20: 1011-1014.

7. Kimura J. Electrodiagnosis in diseases of nerve and muscle: principles and practices. Philadelphia: FA Davis;1983. p 106111.
8. Letz R, Gerr F. Covariates of human peripheral nerve function: I. Nerve conduction velocity and amplitude. Neurotoxicol Teratol 1994;16:95-104.

9. Nathan PA, Keniston RC, Myers LD, Meadows KD. Obesity as a risk factor for slowing of sensory conduction of the median nerve in industry: a cross-sectional and longitudinal study involving 429 workers. J Occup Med 1992;34:379-383.

10. Nordstrom DL, Vierkant RA, DeStefano F, Layde PM. Risk factors for carpal tunnel syndrome in a general population. Occup Environ Med 1997;54:734-740.

11. Ochoa J, Fowler TJ, Gilliatt RW. Anatomical changes in peripheral nerve lesions compressed by a pneumatic tourniquet. J Anat 1972;113:433-455.

12. Radecki P. A gender-specific wrist ratio and likelihood of a median nerve abnormality at the carpal tunnel. Am J Phys Med Rehabil 1994;73:157-162.

13. Vessey MP, Villard-Mackintosh L, Yeates D. Epidemiology of carpal tunnel syndrome in women of childbearing age. Findings in a large cohort study. Int J Epidemiol 1990;19:655-659.

14. Werner CO, Elmqvist D, Ohlin P. Pressure and nerve lesion in the carpal tunnel. Acta Orthop Scand 1983;54:312-316.

15. Werner RA, Albers JW, Franzblau A, Armstrong TJ. The relationship between body mass index and the diagnosis of carpal tunnel syndrome. Muscle Nerve 1994;17:632-636.

16. Werner RA, Albers JW, Franzblau A, Armstrong TJ. The influence of body mass index and work activity in determining the prevalence of median mononeuropathy at the wrist. J Occup Environ Med 1997;54:268-271.

17. Werner RA, Armstrong TJ. Carpal tunnel syndrome: ergonomic risk factors and intra carpal canal pressure. Phys Med Rehabil Clin N Am 1997;8:555-569. 